\title{
2 Pedunculopontine nucleus microelectrode recordings 3 in movement disorder patients
}

\author{
4 Moran Weinberger - Clement Hamani · \\ 5 William D. Hutchison • Elena Moro • \\ 6 Andres M. Lozano · Jonathan O. Dostrovsky \\ 7 Received: 29 October 2007 / Accepted: 6 March 2008 \\ 8 (C) Springer-Verlag 2008
}

\begin{abstract}
The pedunculopontine nucleus (PPN) lies within the brainstem reticular formation and is involved in the motor control of gait and posture. Interest has focused recently on the PPN as a target for implantation of chronic deep brain stimulation (DBS) electrodes for Parkinson's disease (PD) and progressive supranuclear palsy (PSP) therapy. The aim of this study was to examine the neurophysiology of the human PPN region and to identify neurophysiological landmarks that may aid the proper placement of DBS electrodes in the nucleus for the treatment of PD and PSP. Neuronal firing and local field potentials were recorded simultaneously from two independently driven microelectrodes during stereotactic neurosurgery for implantation of a unilateral DBS electrode in the PPN in five PD patients and two PSP patients. Within the PPN region, the majority $(57 \%)$ of the neurons fired randomly while about $21 \%$ of the neurons exhibited 'bursty' firing. In addition, $21 \%$ of the neurons had a long action potential duration and significantly lower firing rate suggesting they were cholinergic neurons. A change in firing rate produced by passive and/or active contralateral limb movement was
\end{abstract}

M. Weinberger · W. D. Hutchison · J. O. Dostrovsky $(\bowtie)$ Department of Physiology, University of Toronto, Med Sci Bldg 3302, 1 King's College Circle,

M5S 1A8 Toronto, ON, Canada

e-mail: j.dostrovsky@utoronto.ca

M. Weinberger

e-mail: moran.weinberger@utoronto.ca

C. Hamani - W. D. Hutchison - E. Moro - A. M. Lozano ·

J. O. Dostrovsky

Toronto Western Research Institute, Toronto, ON, Canada

C. Hamani · E. Moro · A. M. Lozano

Toronto Western Hospital, Division of Neurosurgery,

University of Toronto, Toronto, ON, Canada observed in $38 \%$ of the neurons that were tested in the PPN region. Interestingly, oscillatory local field potential activity in the beta frequency range $(\sim 25 \mathrm{~Hz})$ was also observed in the PPN region. These electrophysiological characteristics of the PPN region provide further support for the proposed role of this region in motor control. It remains to be seen to what extent the physiological characteristics of the neurons and the stimulation-evoked effects will permit reliable identification of PPN and determination of the optimal target for DBS therapy.

Keywords Pedunculopontine tegmental nucleus · Parkinson disease · Progressive supranuclear palsy • Deep brain stimulation $\cdot$ Basal ganglia

\section{Introduction}

Although STN deep brain stimulation (DBS) to treat advanced Parkinson's disease (PD) is a well established procedure and produces striking clinical improvement in motor symptoms such as tremor, rigidity and bradykinesia, it has only a moderate and a short term effect in improving axial symptoms like gait akinesia and postural instability (Kleiner-Fisman et al. 2003). Recently, the pedunculopontine tegmental nucleus (PPN) has been proposed (Pahapill and Lozano 2000) and examined as an alternative therapeutic target for PD, especially for patients with severe gait and postural impairment (Mazzone et al. 2005; Plaha and Gill 2005; Stefani et al. 2007). The PPN is part of the brainstem reticular formation and projects to the thalamus and spinal cord (Erro and Gimenez-Amaya 1999). It is also reciprocally connected to the basal ganglia and is believed to regulate the control of complex central nervous system functions including posture and gait (for review, see Pahapill and
30 31 32 33 34 35 36 37 38

\begin{tabular}{|l|l|c|l|lcl|}
\hline & Large 221 & 1349 & xxxx & Dispatch: 10.3.08 & \multicolumn{2}{l|}{ No. of Pages: 10 } \\
\cline { 1 - 4 } & Article & MS Code & LE $\square$ & TYPESET $\square$ & CP $\square$ & DISK $\square$ \\
\hline
\end{tabular}


Lozano 2000). In primates, the PPN receives a major projection from the two output structures of the basal ganglia (substantia nigra pars reticulata and internal globus pallidus-GPi) and the subthalamic nucleus (STN). In turn, the PPN projects to the substantia nigra pars compacta and STN (Garcia-Rill 1991; Pahapill and Lozano 2000; Winn 2006). This organization emphasizes the potential role of the PPN in controlling basal ganglia output through subsidiary loops.

It has been shown that neurons in the PPN region degenerate in akinetic disorders such as $\mathrm{PD}$ and progressive supranuclear palsy (PSP) (Hirsch et al. 1987; Jellinger 1988; Zweig et al. 1989), and lesions in the PPN can lead to decreased movements (Aziz et al. 1998; Kojima et al. 1997). Therefore, it has been suggested that the PPN may be an alternative target for DBS for the treatment of PD (Jenkinson et al. 2004; Matsumura 2005; Pahapill and Lozano 2000; Plaha and Gill 2005). Indeed, recent studies have shown that low frequency stimulation $(<25 \mathrm{~Hz})$ of the PPN increases motor activity in a monkey model of PD (Jenkinson et al. 2004) and improves clinical motor scores in PD patients (Mazzone et al. 2005; Plaha and Gill 2005; Stefani et al. 2007).

The PPN occupies an area in the mesopontine tegmentum that contains two populations of neurons, non-cholinergic (likely glutamatergic) and cholinergic (Mesulam et al. 1989). The cholinergic neurons have larger somata, but are less numerous than the non-cholinergic neurons (Honda and Semba 1995; Rye et al. 1987; Spann and Grofova 1992). Electrophysiological studies of the PPN in nonhuman primates have rarely appeared in the literature (Matsumura 2005). In the PPN of the normal monkey, nearly half of the neurons recorded changed their firing rates in response to voluntary movement of the ipsi-and/ or contralateral arm (Matsumura et al. 1997). In the cat it has been shown that PPN neurons change their firing activity during a conditioned movement of the forelimb (Dormont et al. 1998) and that inactivation of the PPN by injection of muscimol (a $\gamma$-aminobutyric acid (GABA) agonist) induces prolonged arrest in the performance of the task (Conde et al. 1998). These findings suggest that the PPN is involved in the initiation and modulation of movements (see review: Matsumura 2005).

The aim of the present study was to describe the neurophysiology of the human PPN region and identify neurophysiological landmarks and characteristics that may help in localizing the target for insertion of DBS electrodes in the nucleus for the treatment of PD and PSP.

Patients

We studied seven patients, five with advanced PD and two with PSP, who were undergoing stereotactic surgery for implantation of unilateral PPN-DBS electrodes under local anesthesia. The group consisted of two women and five men who, at the time of operation, had a mean age $( \pm \mathrm{SD})$ of $64 \pm 6.7$ years and a mean disease duration of $12.4 \pm$ 5.1 years. During surgery the patients were awake and 'off' dopaminergic medications for at least $12 \mathrm{~h}$ from the last oral dose of medication. Patient \#7 had bilateral STN-DBS electrodes that were implanted one year before the surgery and were switched off during the recordings. Demographic details of the patients are given in Table 1 . The study was performed with approval of the University Health Network Ethical Review Board, University of Toronto. Patients gave written and informed consent before surgery.

Surgical procedure and targeting

On the morning of the surgery, a stereotactic frame (Leksell G, Elekta, Inc, Atlanta, Ga) was affixed to the patients'

Table 1 Demographic and clinical features of the patients selected for surgery

\begin{tabular}{|c|c|c|c|c|c|c|c|c|c|c|}
\hline $\begin{array}{l}\text { Patient } \\
\# \text { and } \\
\text { disease }\end{array}$ & $\begin{array}{l}\text { Age } \\
\text { (year) and } \\
\text { gender }\end{array}$ & $\begin{array}{l}\text { Disease } \\
\text { duration } \\
\text { (year) }\end{array}$ & $\begin{array}{l}\text { L-Dopa } \\
\text { therapy } \\
\text { (year) }\end{array}$ & $\begin{array}{l}\text { UPDRS } \\
\text { III (off/on) }\end{array}$ & $\begin{array}{l}\text { Gait } \\
\text { sub-score } \\
\text { (off /on) }\end{array}$ & $\begin{array}{l}\text { Postural } \\
\text { instability } \\
\text { sub-score (off /on) }\end{array}$ & $\begin{array}{l}\text { Freezing } \\
\text { (off/on) }\end{array}$ & $\begin{array}{l}\text { Side } \\
\text { of brain }\end{array}$ & $\begin{array}{l}\text { Span } \\
\text { of record } \\
(\mathrm{mm})\end{array}$ & $\begin{array}{l}\text { Number of } \\
\text { neurons sampled } \\
\text { (PPN/total) }\end{array}$ \\
\hline 1-PD & $63 \mathrm{M}$ & 10 & 5 & $34.5 / 20$ & $2.5 / 0.5$ & $3 / 1$ & Yes/yes & $\mathrm{L}$ & 17 & $12 / 34$ \\
\hline 2-PSP & $67 \mathrm{~F}$ & 5 & N/A & $57.5 /$ (NA) & $3.5 / \mathrm{NA}$ & 4/NA & No /NA & $\mathrm{R}$ & 13 & $7 / 19$ \\
\hline 3-PSP & $70 \mathrm{M}$ & 10 & N/A & $38.5 /(\mathrm{NA})$ & $3.5 / \mathrm{NA}$ & $3.5 / \mathrm{NA}$ & No/NA & $\mathrm{L}$ & 13 & $3 / 9$ \\
\hline 4-PD & $68 \mathrm{M}$ & 15 & 14 & $41 / 21$ & $2.5 / 0.5$ & $2.5 / 1$ & Yes/no & $\mathrm{L}$ & $\begin{array}{l}18 \\
13\end{array}$ & $\begin{array}{l}12 / 45 \\
18 / 35\end{array}$ \\
\hline 5-PD & $67 \mathrm{M}$ & 21 & 15 & $42 / 20$ & $2 / 0.5$ & $2 / 1$ & Yes/no & $\mathrm{L}$ & 16 & $12 / 37$ \\
\hline 6-PD & $63 \mathrm{~F}$ & 11 & 12 & $43.5 / 35$ & $2 / 1.5$ & $2 / 2$ & Yes/no & $\mathrm{R}$ & 13 & $3 / 13$ \\
\hline 7-PD ${ }^{a}$ & $50 \mathrm{M}$ & 15 & 17 & $25.5 / 14$ & $2 / 0.5$ & $0 / 0$ & Yes/no & $\mathrm{R}$ & 14 & $15 / 43$ \\
\hline
\end{tabular}

NA not applicable

a Patient had bilateral STN-DBS

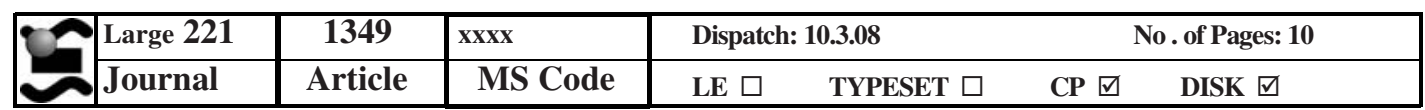


129 heads and preoperative magnetic resonance (MR) images

130 were obtained (Signa, 1.5 T, General Electric, Milwaukee,

131 Wis). To target the PPN we used 3D inversion recovery and

132 T2-weighted axial sequences. Triplanar reconstruction of

133 the images with the axial series parallel to the plane of the

134 anterior (AC) and posterior commissures (PC) was carried

135 out on a surgical neuronavigation workstation (Mach 4.1,

136 StealthStation, Medtronic, SNT). In the dorsal ventral

137 axis, a region that was 4-5 mm below the caudal aspect of

138 the inferior colliculus was targeted on sagittal images. In

139 the anteroposterior axis, our target was $2-3 \mathrm{~mm}$ posterior

140 to the limit between the base and tegmentum of the pons.

141 In the medial lateral axis, we targeted a region that was

142

3-4 $\mathrm{mm}$ medial to the lateral edge of the brainstem.

Once the MRI based coordinates of the target were calculated, the patient was brought to the operating room and a burr hole was drilled $2 \mathrm{~cm}$ from the midline in front of the coronal suture. Microelectrode recordings were then conducted from $15 \mathrm{~mm}$ above the target and extended $2-5 \mathrm{~mm}$ below. In each patient except one, only one dual electrode track (consisting of two microelectrodes $600 \mu \mathrm{m}$ apart-see Levy et al. 2007 for details) was performed. In patient \#4 a second electrode track was performed $2 \mathrm{~mm}$ medial to the first since the thresholds for evoking paresthesia in the first track were very low.

\section{Microelectrode recordings and stimulation}

Neuronal spontaneous firing activity and local field potentials (LFPs) were recorded simultaneously from two independently driven microelectrodes (about $25 \mu \mathrm{m}$ tip length, axes $600 \mu \mathrm{m}$ apart, about $0.2 \mathrm{M} \Omega$ impedance at $1,000 \mathrm{~Hz}$ ) during the electrophysiological mapping procedure used to obtain physiological data for localizing the target for the DBS electrode placement (for further details of the procedure see: Hutchison et al. 1998). All recordings were amplified 5,000-10,000 times and filtered at $10-5,000 \mathrm{~Hz}$ (analog Butterworth filters: high-pass, one pole; low-pass, two poles) using two Guideline System GS3000 (Axon Instruments, Union City, CA) amplifiers. Recorded signals were digitized at $10 \mathrm{kHz}$ with a CED 1401 (Cambridge Electronic Design, Cambridge, UK). In all patients, we tested the effects of a $1 \mathrm{~s}$ microstimulation train $(200 \mathrm{~Hz}$, $150 \mu$ s pulse width at intensities up to $100 \mu \mathrm{A}$ ) along the microelectrode trajectory at intervals of $2 \mathrm{~mm}$ or less.

\section{Localization of electrode trajectory}

In the present study, the approximate location of the microelectrode tracks was estimated as follows: Preoperative axial stereotactic 3D inversion recovery and T2-weighted MR images were transferred to a StealthStation workstation. Using the FrameLink 4.1 software (Mach 4.1, StealthStation,
Medtronic, SNT) these two images were merged, the fiducials of the frame were recognized, and the mean rod marking error was calculated and registered. Coronal and sagittal planes were reconstructed based on axial images. The AC and PC were then targeted in the axial plane and three additional points were plotted in the midline. Thereafter, the images were reformatted parallel to the AC-PC plane and orthogonal to the midline. Pitch, roll, and yaw were corrected in the StealthStation. The coordinate of the microelectrode track and the angles of each trajectory were entered into the neuronavigation system so that they could be reconstructed. As the PPN cannot be clearly visualized on imaging studies, we estimated the location of the region that most likely encompassed the nucleus. We defined this PPN region as the anterolateral portion of the pontine/ mesencephalic tegmentum that extended from $2 \mathrm{~mm}$ below the inferior colliculus to the transition between the inferior and superior colliculi, according to the Paxinos and Huang atlas (Paxinos and Huang 1995). The coordinates chosen for electrophysiological recordings were similar across patients.

Data analysis

Only sites with good signal-to-noise and stable single unit recordings were analyzed. To characterize the firing activity of the recorded neurons the signals were bandpass filtered at $425-5,000 \mathrm{~Hz}$ and single unit activity was discriminated using the template matching tool in Spike2 (CED). Mean firing rates and measurement of firing patterns were obtained using a modification of the Kaneoke and Vitek method (Kaneoke and Vitek 1996), which uses discharge density to categorize firing patterns into random, bursty or regular. As a measure of action potential width we used the duration of the negative phase of the averaged spike waveform (see Fig. 1a). A similar method was previously used to measure spike duration of PPN neurons by Matsumura et al. (1997) and Takakusaki et al. (1997).

For examining neuronal responses to movement, movement onset was determined from accelerometer recordings and/or electromyograms and a peri-stimulus histogram constructed. Movement-related changes in neuronal firing were initially identified by visual inspection of the histograms. Then, the identified responses were evaluated statistically using nonparametric tests comparing the firing rate during a 200 or $300 \mathrm{~ms}$ control period and during 200/300 ms of the visually determined response $(P<0.05$, Wilcoxon signed rank test).

For spectral analysis, spike times and unfiltered LFP data were imported into MATLAB (version 6.5, The Math Works, Natick, MA). Only data of $\geq 11 \mathrm{~s}$ duration (mean \pm SD: $37.9 \pm 19.7$ s, range: $11-112 \mathrm{~s}$ ) were analyzed. Spectral analysis of neuronal discharges and LFP recordings

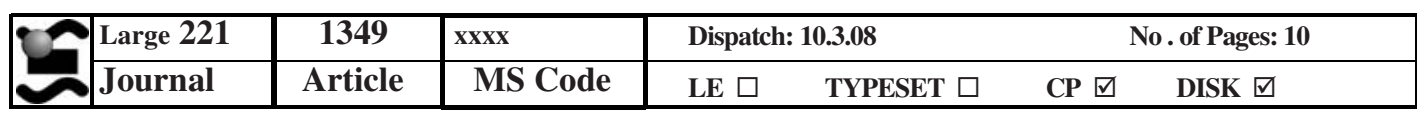


Fig. 1 Width of action potentials. (a) The width of the action potential was measured as the duration of the negative phase of the averaged spike waveform (b) Distribution histogram of spike durations
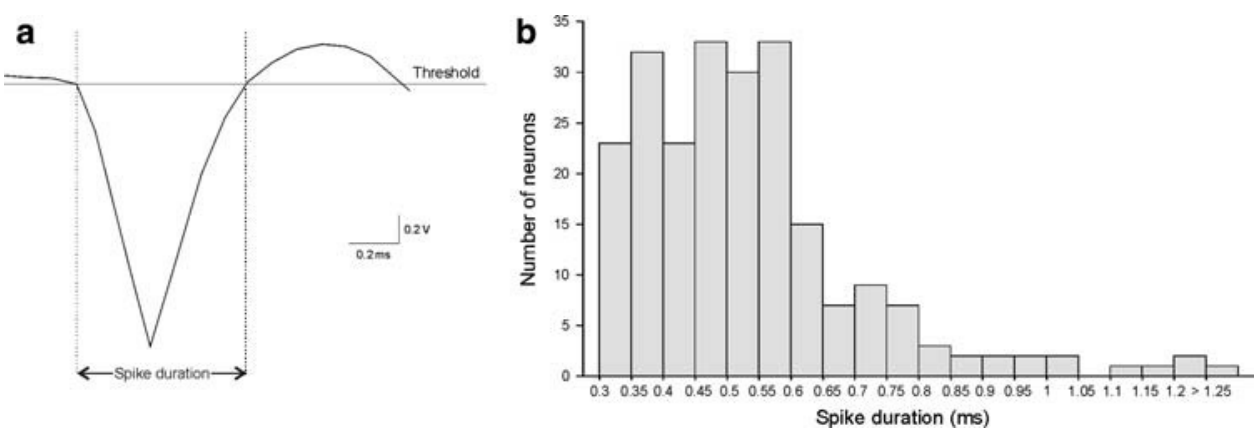

was performed according to Halliday et al. (1995). After signals were down-sampled to $1 \mathrm{kHz}$, spectra of LFP power were estimated by dividing the waveform signal into a number of sections of equal duration of $1.024 \mathrm{~s}(1,024$ data points, 512 point overlap), each section was windowed (Hanning window) and the magnitude of the 1,024 discrete Fourier transforms of each section was squared and averaged to form the power spectrum for frequencies up to $80 \mathrm{~Hz}$ with a frequency resolution of $0.97 \mathrm{~Hz}$. The power was transformed to a logarithmic scale and shown in decibels $(\mathrm{dB})$. Since the estimated power spectrum has a distribution which is analogous to a $\chi^{2}$ distribution, the $95 \%$ confidence intervals were given on the basis of the $\chi^{2}$ distribution (Jarvis and Mitra 2001) while the degrees of freedom is based on the number of windowed sections. For detecting significant neuronal oscillations we used a process of spike train shuffling (see Rivlin-Etzion et al. 2006).

Coherence and cross-correlation analysis (Halliday et al. 1995; Rosenberg et al. 1989) was used to assess the relationship between simultaneously recorded data from separate electrodes and between LFP and spike data recorded from the same microelectrode. The coherence function provides a frequency domain bounded measure of association, taking on values between 0 and 1 , with 0 in case of independence and 1 in case of a perfect linear relationship of both frequency, amplitude and phase. The $95 \%$ confidence level is given by $1-(0.05)^{1 /(\mathrm{L}-1)}$, where $L$ is the number of disjoint sections. Cross-correlation provides a time-domain measure of dependency between random processes. Under the hypothesis that in the case of two independent processes the value of the cross-correlation function is zero, the upper and lower $95 \%$ confidence limits for the estimated crosscorrelation are given by $0 \pm 1.96$ (variance $\left._{\text {cross-corr }}\right)^{1 / 2}$.

\section{Results}

Action potentials and firing properties

A total of 244 neurons was recorded in seven patients. Of these, 235 were recorded while the patient was at rest and were used to characterize the baseline firing activity. Neu- rons were classified according to the width and polarity of their action potentials. The incidence histogram of spike durations did not reveal a clear bimodal distribution. Nevertheless, we observed a marked drop in the number of neurons that had action potential durations $\geq 0.65 \mathrm{~ms}$ (see Fig. 1b), which suggests they may have originated from a different population of neurons that are likely to be composed of cholinergic neurons (Dormont et al. 1998; Takakusaki et al. 1997). This population consisted of 38 neurons that comprised $15.6 \%$ of the total number of neurons. These neurons were found in all the patients except one (patient \#3, PSP) in whom a total of only nine neurons were recorded (see Table 1$)$. Their mean $( \pm \mathrm{SD})$ spike duration was $0.84 \pm 0.2 \mathrm{~ms}$ compared with $0.47 \pm 0.1 \mathrm{~ms}$ for the neurons with brief spikes. In addition, these neurons had a significantly lower mean firing rate (medians: 9.3 vs. 17.3 spikes/s, $P \leq 0.001$, Mann-Whitney rank sum test) (Fig. 2b, d). This provides further support for the assumption that these cells represent a different neuronal population. The majority of these neurons (86.8\%) was found within and below the PPN region. In the region of the PPN, these neurons comprised $21 \%$ of the neurons $(18 / 85)$ compared to $19.5 \%$ below that region (15/77) and $6 \%$ above (5/82).

Interestingly, 16 units had positive-going action potentials and significantly higher mean firing rates (median 67.6 spikes/s, $P \leq 0.001$ ) (Fig. 2c, d). These units were found in all seven patients. Six of these neurons were located in the PPN region and nine were located below that region. In addition, most (88\%) of these neurons displayed a regular firing pattern as determined by the Kaneoke and Vitek method (Fig. 3a). This was significantly different from the patterns of activity exhibited by the two types of neurons described above which were primarily random (65 and $68 \%$ for the neurons with short and long spike durations respectively) $\left(P \leq 0.001, \chi^{2}\right.$-test). Comparison of the proportions of both brief-and broad-spike duration neurons exhibiting regular, random or bursty firing patterns above, within and below the PPN region, revealed a statistically significant difference $\left(P=0.016, \chi^{2}\right.$-test) (Fig. $\left.3 b\right)$. In the PPN region, the majority $(57 \%)$ of the neurons fired randomly while about $21 \%$ of the neurons exhibited 'bursty' firing. Above and below the PPN region, the proportion of

\begin{tabular}{|c|c|c|c|c|c|c|}
\hline Large 221 & 1349 & $\mathbf{x x x x}$ & Dispatc & 0.3 .08 & & of Pages: 10 \\
\hline Journal & Article & MS Code & LE $\square$ & TYPESET $\square$ & CP $\square$ & DISK ఐ \\
\hline
\end{tabular}


Fig. 2 Classification into three groups of neurons and their mean firing rates. $(\mathbf{a}-\mathbf{c})$ Superimposition of action potential neurons displaying different spike durations and polarities (top trace) with $2 \mathrm{~s}$ of their spontaneous firing bandpass filtered at $425-5,000 \mathrm{~Hz}$ (bottom trace). a short duration negative spikes; b long duration negative spikes; c positive spikes. d Box plot of firing rates of the three types of neurons. The boxes represent the span of the first to third interquartile range in data distribution and the filled dots depict the outliers. The median is marked by a horizontal line waveforms obtained from three
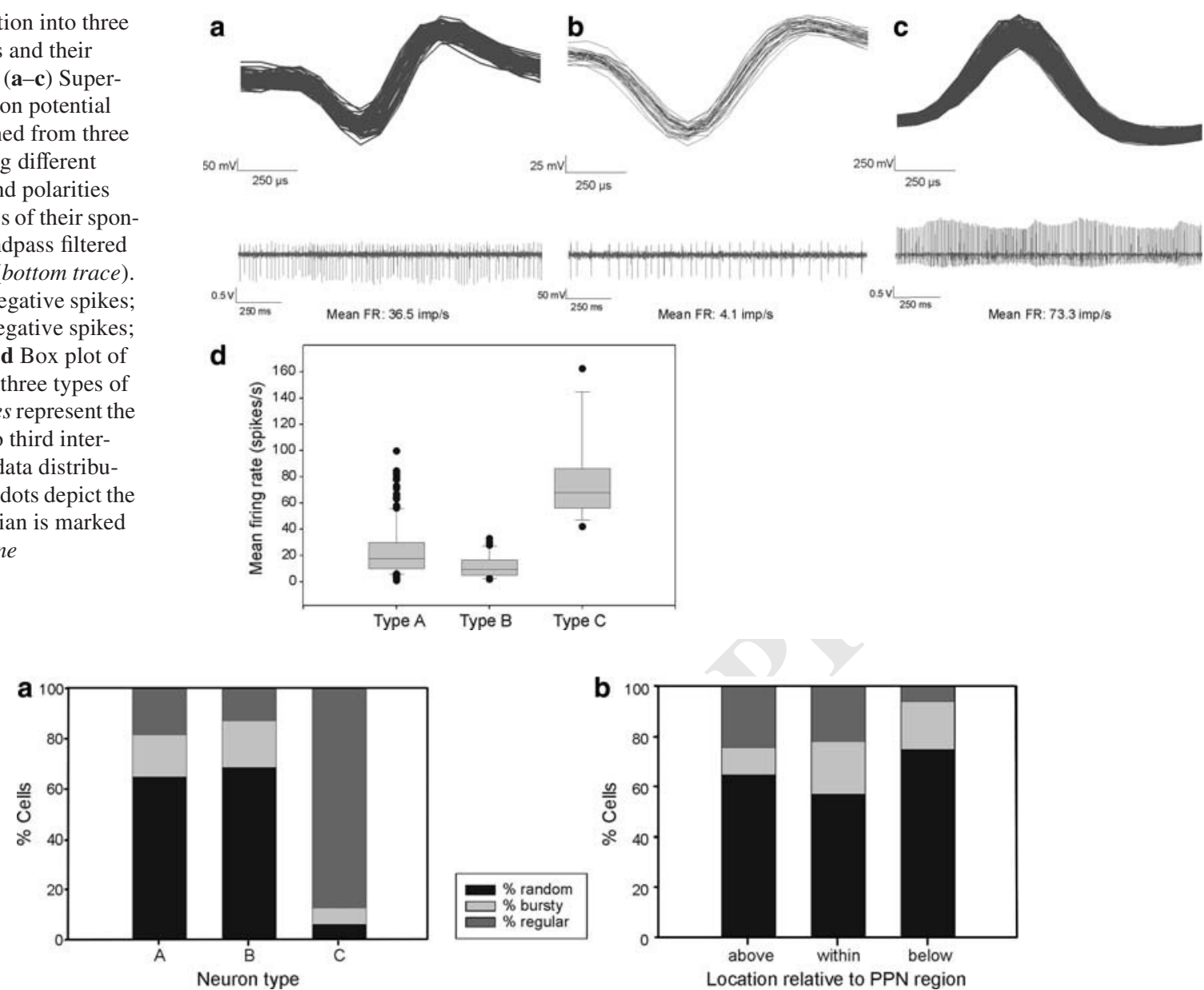

Fig. 3 Comparison of proportions of firing patterns for each cell type and location. a Stacked bar graphs showing percentage of neurons in each firing pattern category for each of the three types of neurons $\left(P \leq 0.001, \chi^{2}\right.$-test). b Stacked bar graphs showing percentage of neu-

rons in each firing pattern category for both brief- and broad-spike duration neurons according to their location relative to the PPN region $\left(P=0.016, \chi^{2}\right.$-test $)$

309 bursting neurons was smaller (11 and 19\% respectively) 310 while more neurons exhibited random firing (65 and 75\%

311 respectively).

312 Neurons in the PPN region were responsive to movements

313 The activity of 103 neurons was recorded during contralat-

314 eral limb movements. Movement tasks varied between pas-

315 sive and/or voluntary movements of the wrist, hand, foot,

316 elbow and sometimes shoulder. $36 \%(n=37)$ of the neurons

317 responded to at least one type of passive or voluntary

movement of the limb. Out of the 41 neurons that were 318 tested only for passive movements, 16 responded $(39 \%)$ and out of the 50 neurons that were tested only for voluntary movements, 12 responded (24\%). Twelve neurons were tested for both passive and voluntary movement, out of which, five neurons responded to passive movement only, two neurons responded to voluntary movement only and two neurons responded to both. Table 2 summarizes the results for each of the neuron types. The changes in firing rates were mostly excitatory (about 80\%) and in some cases, were followed by inhibition (see Fig. 4a, c). Within
Table 2 Neuron types and movement-related activity

Note that 12 neurons that were tested for both passive and voluntary movement are included in both groups

$\mathrm{Nr}$ no response

\begin{tabular}{|c|c|c|c|c|c|c|c|c|c|}
\hline \multirow{2}{*}{$\begin{array}{l}\text { Neuron } \\
\text { type }\end{array}$} & \multicolumn{4}{|c|}{ Passive movement } & \multicolumn{4}{|c|}{ Voluntary movement } & \multirow[t]{2}{*}{ All } \\
\hline & Increase & Decrease & $\mathrm{Nr}$ & Total & Increase & Decrease & $\mathrm{Nr}$ & Total & \\
\hline A & 17 & 4 & 25 & 46 & 11 & 2 & 37 & 50 & 96 \\
\hline B & 0 & 1 & 5 & 6 & 1 & 1 & 7 & 9 & 15 \\
\hline $\mathrm{C}$ & 1 & 0 & 0 & 1 & 1 & 0 & 2 & 3 & 4 \\
\hline Total & 18 & 5 & 30 & 53 & 13 & 3 & 42 & 62 & 115 \\
\hline
\end{tabular}

\begin{tabular}{|c|c|c|c|c|c|c|}
\hline Large 221 & 1349 & $\mathbf{x x x x}$ & Dispatc & 0.3 .08 & & of Pages: 10 \\
\hline Journal & Article & MS Code & LE $\square$ & TYPESET $\square$ & CP $\square$ & DISK $\square$ \\
\hline
\end{tabular}


Fig. 4 Examples of movementevoked responses of the three types of neurons. a An example of a type A neuron exhibiting an increase in firing rate in response to passive wrist flexion that is followed by a decrease in firing rate $(P=0.001$, Wilcoxon signed rank test). b Type B neuron exhibiting increased firing rate to passive elbow movement $(P \leq 0.001)$. c Type $\mathrm{C}$ neuron exhibiting increased firing followed by decreased firing in response to passive flexion and extension of the foot respectively $(P=0.003)$. All movements were contralateral to the recording side. Raster plots (middle traces) and stimulus histograms (bottom traces) were aligned to movement onset (indicated by smoothed and rectified EMG (a, b) or accelerometer (c) output) (top traces) $\mathbf{a} \underset{\Phi}{\mathbf{\Phi}}$
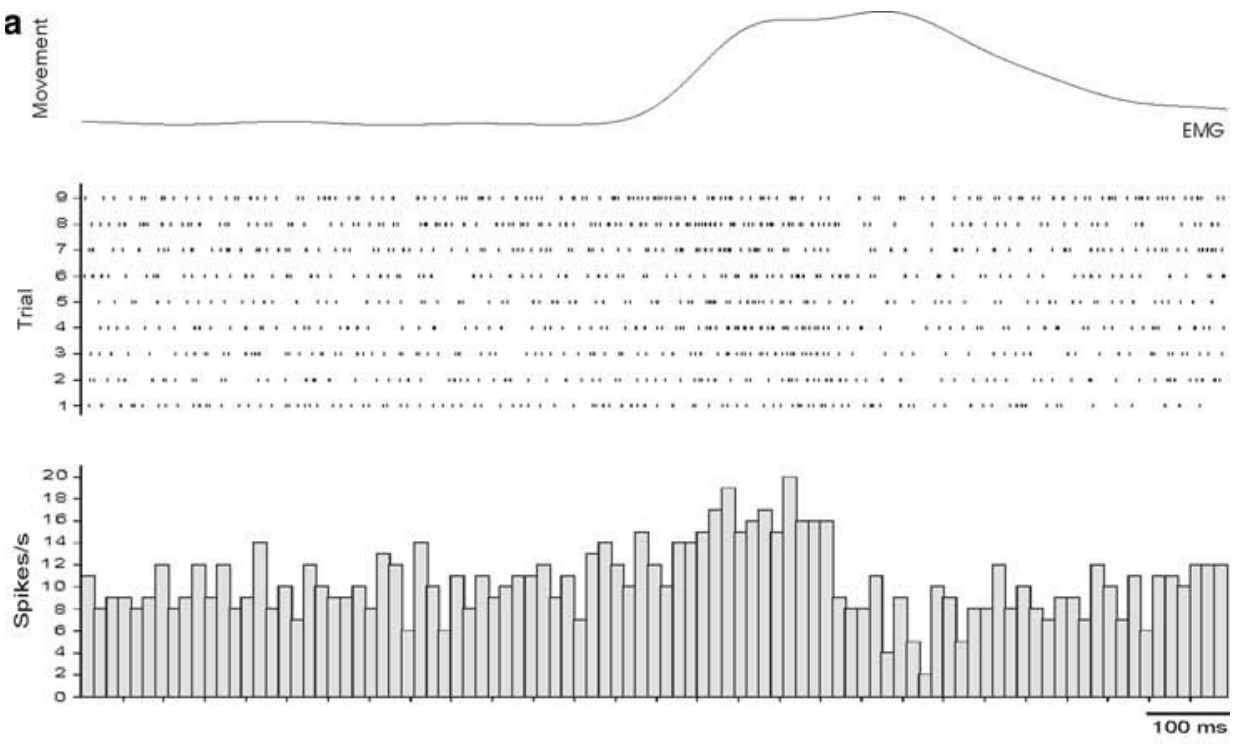

b
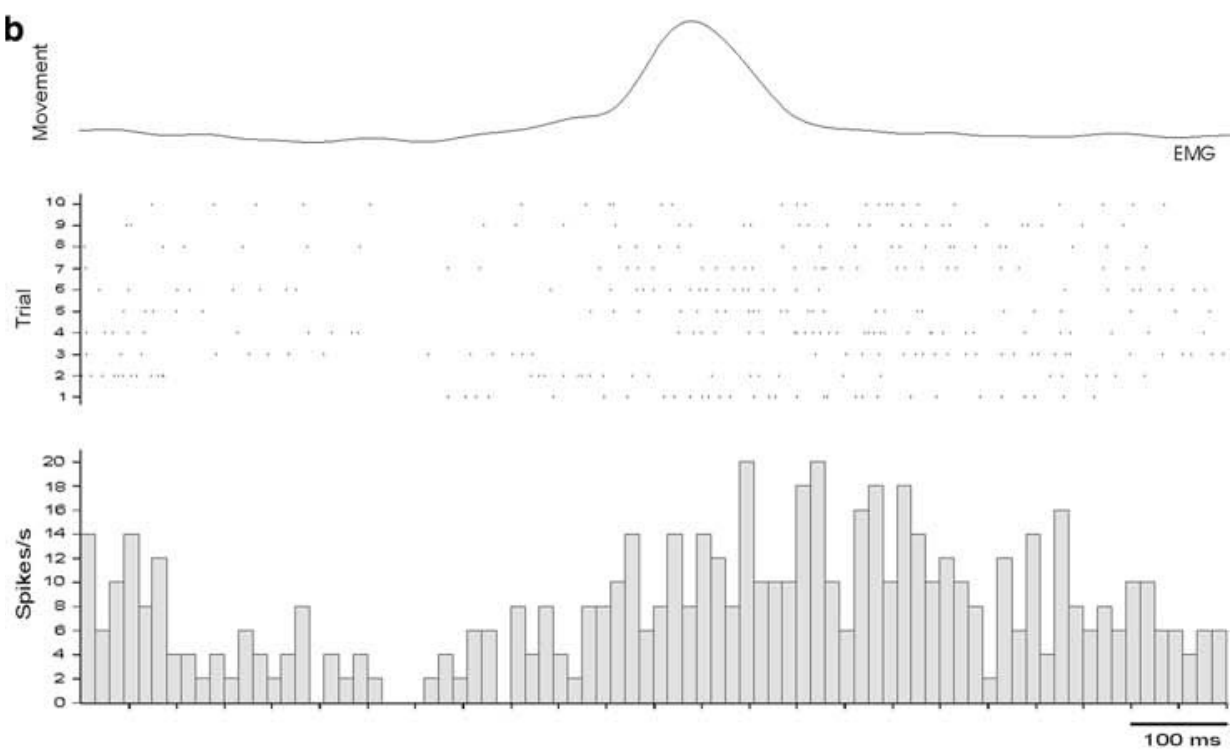

C

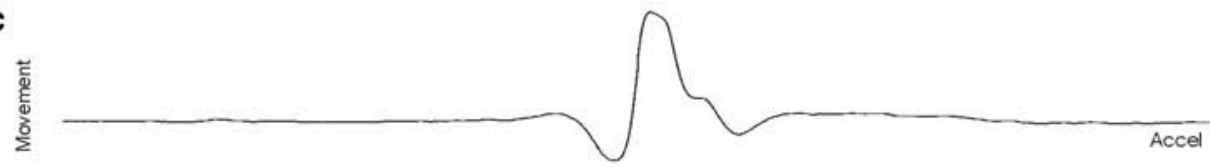

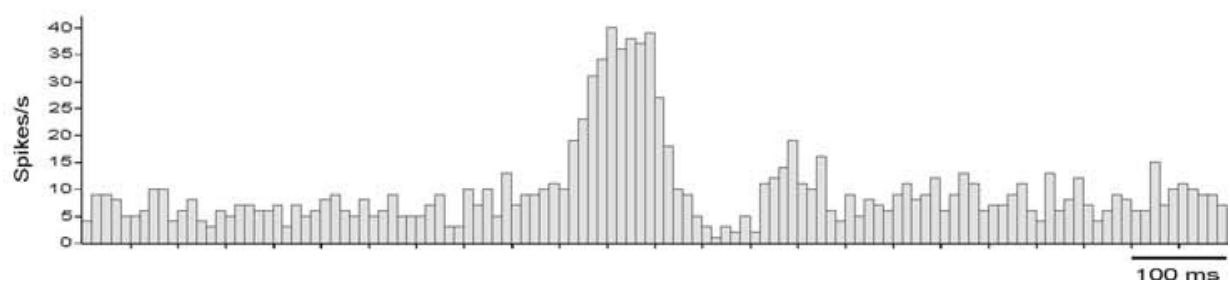

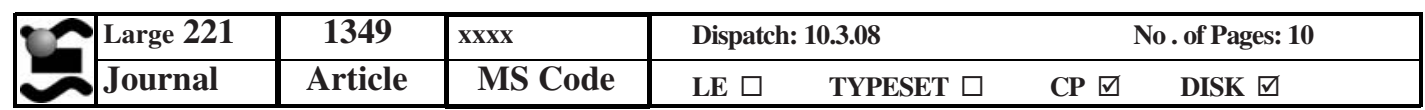


329 the PPN region $38 \%$ of the neurons responded to movement 330 and similar percentages were recorded above and below the 331 PPN region where 37 and $33 \%$ of the neurons responded 332 respectively. Movement responsive neurons included all 333 three neuron types. Figure 4 shows an example of 334 responses to contralateral movement from each neuron 335 type.

337 In all patients, $1 \mathrm{~s}$ stimulus trains evoked paresthesia at 338 some sites along the microelectrode trajectory. In three 339 patients, the threshold intensities for stimulation-evoked 340 paresthesia varied between 50 and $100 \mu \mathrm{A}$ (with three 341 exceptions of 20,25 and $25 \mu \mathrm{A}$ ) (mean \pm SD: $79.2 \pm$ $34229.6 \mu \mathrm{A}, n=24)$. In the remaining four patients, the threshold 343 intensities were lower and varied between 1 and $50 \mu \mathrm{A}$ 344 (with two exceptions of 100 and $75 \mu \mathrm{A}$ ) (mean \pm SD: $34518.8 \pm 19.9 \mu \mathrm{A}, n=55)$. The thresholds were not signifi346 cantly different when stimulating within or close to the 347 PPN region. These responses were assumed to be due to 348 activation of ascending axons in the medial lemniscus. In 349 addition, eye movements were evoked by microstimulation 350 in two patients and occurred only at the maximum stimula351 tion intensity of $100 \mu \mathrm{A}$, and only within and up to $2 \mathrm{~mm}$ 352 above the PPN region.
No obvious differences were noted in any of the findings between the two groups of patients other than a lower number of neurons encountered in the PSP patients compared to all but one of the PD patients.

\section{Discussion}

The present study provides new data documenting various electrophysiological characteristics of the PPN region in PD and PSP patients. The lower number of cells recorded from the PPN region in the two PSP patients (see Table 1) suggests a lower density of neurons which might reflect the large degree of neuronal degeneration and brainstem atrophy that occurs in PSP (Dickson et al. 2007; Hirsch et al. 1987). We did not observe any obvious neurophysiological differences between the two patient groups. However, in view of the small number of neurons in the 2 PSP patients this comparison may not be very meaningful.

Analysis of spike duration and shape revealed three types of neurons. The existence of short and long duration negative going action potentials in this region agrees with the findings of previous in vitro and in vivo animal studies. In vivo recordings from the PPN in rats (Takakusaki et al. 1997), cats (Dormont et al. 1998) and monkeys (Matsumura et al. 1997) revealed the existence of two types of neurons: ones which fire at low rate and exhibit a long duration spike, and ones which fire at higher rates and exhibit a short duration spike. Yet, the incidence histogram of spike durations in our study did not reveal a clear bimodal distribution as previously demonstrated in the rat PPN (Takakusaki et al. 1997). That study, however, utilized intracellular recordings and therefore cannot be directly compared to our extracellular recordings. Moreover, the incidence histogram in our study is consistent with data from extracellular recordings in the monkey PPN that failed to exhibit a bimodal distribution (Matsumura et al. 1997).

It has been proposed on the basis of in vitro studies that the neurons with broad spikes are cholinergic (Dormont et al. 1998; Takakusaki et al. 1997). Although the exact borders of the PPN remain indistinct, the cholinergic neurons are a very obvious component of the PPN (Winn 2006). Indeed, the neurons with broad spikes in our study were found primarily within the region of the PPN and below it. The region below the PPN is likely to be the adjacent laterodorsal tegmental nucleus which is known to contain cholinergic neurons that are interconnected with the cholinergic population in the PPN (Winn 2006). These findings, together with the low rate of firing, provide further support for the assumption that these neurons are cholinergic. In our study, the majority of the neurons in the PPN
384 385 386 387 388 389 390 391 392 393 394

\begin{tabular}{|c|c|c|c|c|}
\hline \begin{tabular}{l|l|} 
Large 221 \\
\end{tabular} & 1349 & $\mathrm{xxxx}$ & Dispatch: 10.3.08 & No . of Pages: 10 \\
\hline Journal & Article & MS Code & TYPESET $\square$ & DISK $\square$ \\
\hline
\end{tabular}


Fig. 5 Beta oscillatory activity in the PPN region. a An example of power spectra of the LFPs recorded from each of the two adjacent microelectrodes (upper and middle traces) and the corresponding coherence function (lower trace). Dotted lines represent $95 \%$ confidence limits. b An example of LFP power changes along the microelectrode track, above within and below the PPN region. The LFP power was normalized and expressed as the percentage of the total power between 5 and $50 \mathrm{~Hz}$

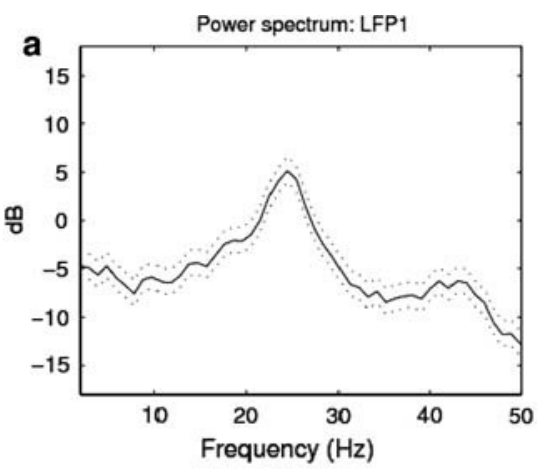

b 10
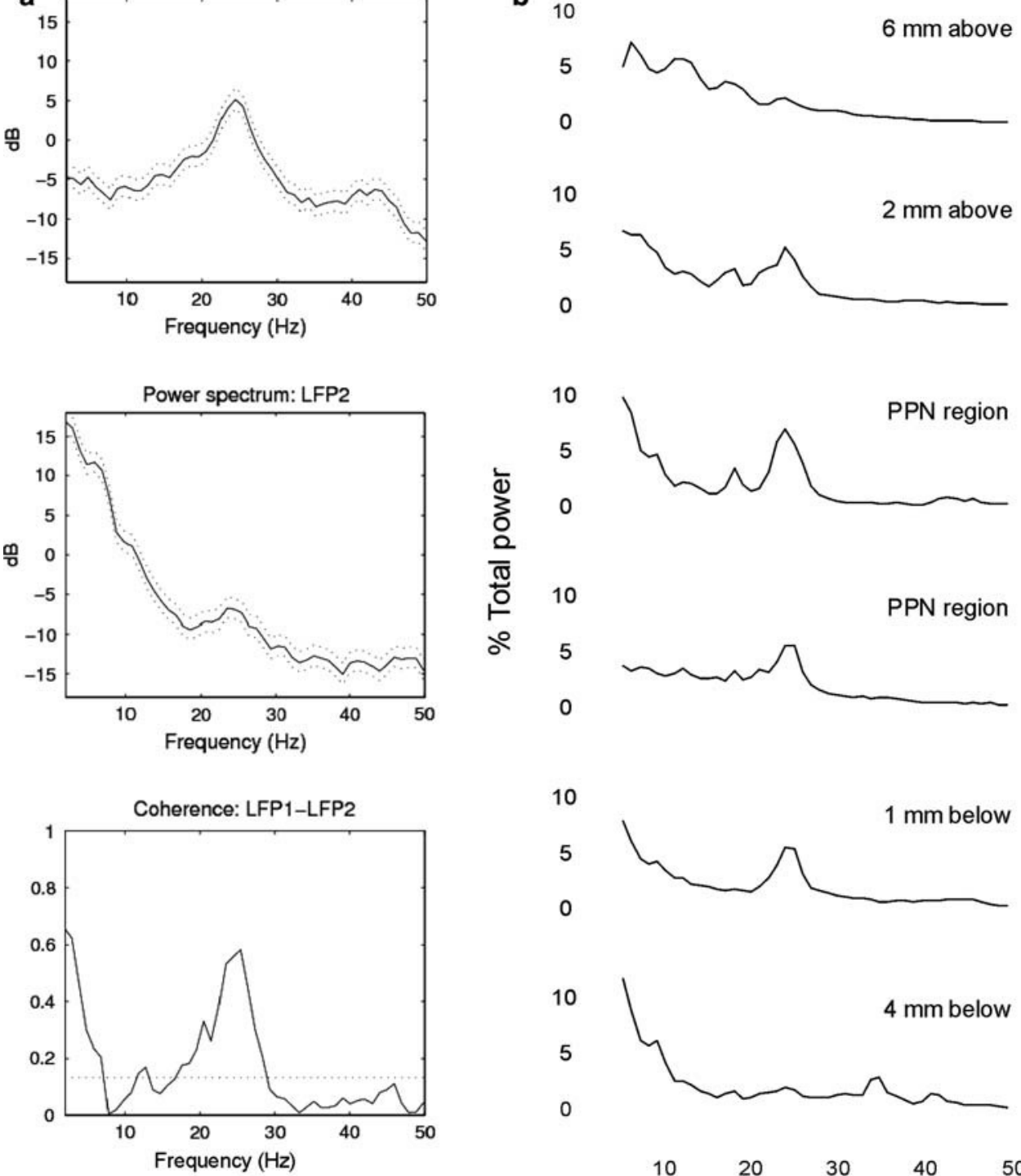

10

5

0
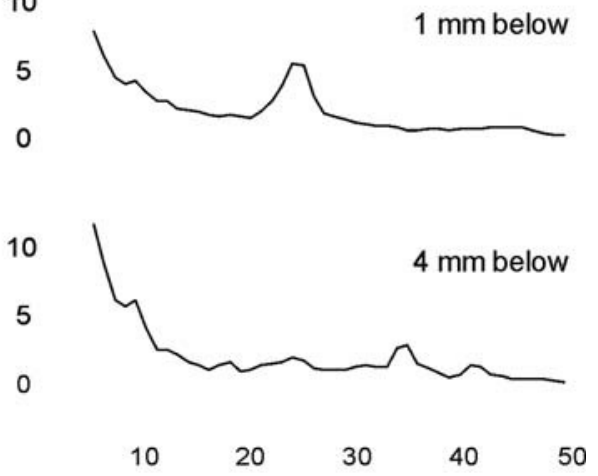

Frequency $(\mathrm{Hz})$ region exhibited random firing while about $21 \%$ of the neurons had a 'bursty' firing pattern. This finding is supported by earlier studies in rats (Scarnati et al. 1987), cats (GarciaRill et al. 2004) and monkeys (Matsumura et al. 1997) which describe a small population of bursty PPN neurons, whereas the majority of the neurons have been reported to fire in an irregular pattern.

The positive-going action potentials may possibly have originated from large diameter axons of passage as extracellularly recorded positive going potentials are generally assumed to be axonal. They had a significantly higher mean firing rate and a regular firing pattern. These electrophysiological properties are different than those of the PPN neurons previously described and are more similar to those seen in the GPi in PD patients (Hutchison et al. 2003; Starr et al. 2005; Tang et al. 2005). Therefore, the positive-going action potentials might be from GPi afferents to the PPN region (Shink et al. 1997) or perhaps from ascending sensory axons of the medial lemniscus which lies laterally to the PPN (Olszewsky and Baxter 1982).

Previous electrophysiological studies in cats and monkeys have identified PPN neurons that exhibit changes in firing rate in response to limb movement and include neurons with both brief and broad spikes (Dormont et al. 1998; Matsumura et al. 1997). In monkey, PPN neurons have also been found to respond with either an increase or a decrease in firing rate to voluntary saccades (Kobayashi et al. 2002). Mazzone et al. (2005) reported finding 6 neurons (of a total of 27 studied) in the PPN of two PD patients that responded with a "slight inhibition or inhibition followed by excitation" to a single-joint movement, but no further details were provided (Mazzone et al. 2005). It is important to note
443 444 445 446 447 448 449 450 451 452 453 454 455 456 457 458

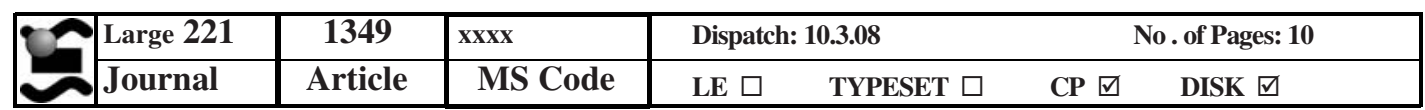


that the PPN region targeted in the current study (PPTg according to Paxinos and Huang 1995) may be different from the region studied by Mazzone et al. (see also Yelnik 2007; Zrinzo et al. 2007a, b). Our study clearly demonstrates the existence of movement-responsive neurons in the human PPN, consistent with the findings of animal studies. Moreover, we found that both short and long spike duration neurons responded to movements. It has been reported that PPN inputs to the basal ganglia are mediated by both cholinergic and glutamatergic synapses (Lavoie and Parent 1994). Taken together, these findings suggest that both groups of neurons may contribute to basal ganglia-related motor functions.

In the three PD patients analyzed, the LFPs in the PPN region exhibited oscillatory activity in the beta range (15$30 \mathrm{~Hz}$ ). Similar oscillations have been previously observed in the STN and GPi of PD patients (Brown et al. 2001; Kuhn et al. 2005; Levy et al. 2002; Weinberger et al. 2006) and are suggested to play an 'antikinetic' role in PD (Brown 2003). It is important to note, however, that the neuronal firing did not show oscillations in these frequencies. The absence of oscillatory firing may be due to limited sampling or may reflect a lack of coupling between dendritic membrane potential oscillations and the soma. Since the beta oscillations were maximal in the PPN region they are unlikely to reflect far field potentials from distant structures (e.g. STN, cortex). The elevation of the beta frequency band LFP activity around the region of the PPN suggests that this activity may be useful in determining the final positioning of the DBS electrode intra-operatively, but further studies are required to examine the consistency of this feature. In a recent study by Androulidakis et al. (2008), LFP recordings from DBS electrodes in the PPN region in six PD patients revealed $7-11 \mathrm{~Hz}$ oscillations that were prominent only after treatment with levodopa, suggesting that these oscillations may represent a physiological pattern of activity in this region (Androulidakis et al. 2008). However, inconsistent with our finding, that study did not reveal beta oscillations in the 'off' dopaminergic state. This discrepancy might be due to the difference in the region targeted in that study, which is similar to that reported by Mazzone et al. (see above).

In summary, the electrophysiological characteristics of the PPN region described in this paper provide further support for the proposed role of this region in motor control. However, in contrast to microelectrode recordings in thalamus, pallidum and STN, we did not find distinctive and definitive neurophysiological characteristics and clear nuclear boundaries for PPN. This is consistent with the absence of precise anatomical borders to the PPN (Winn 2006). Thus microelectrode recordings and microstimulation-induced effects in PPN, although helpful, do not provide the same degree of confirmation of the target as they do in these other regions although future studies may reveal additional features distinctive for the PPN which would be useful in localizing the region and identifying an optimal target for DBS therapy.

Acknowledgments We wish to thank all the patients who participated in this study and Yu Yan Poon for her help with acquiring clinical data. The work was supported by the Canadian Institutes of Health Research grant to JOD (MOP-42505). AML holds a Tier 1 Canada Research Chair in Neurosciences.

\section{References}

Androulidakis AG, Mazzone P, Litvak V, Penny W, Dileone M, Doyle Gaynor LM, Tisch S, Di Lazaaro V, Brown P (2008) Oscillatory activity in the pedunculopontine area of patients with Parkinson's disease. Exp Neurol. in press

Aziz TZ, Davies L, Stein J, France S (1998) The role of descending basal ganglia connections to the brain stem in parkinsonian akinesia. Br J Neurosurg 12:245-249

Brown P (2003) Oscillatory nature of human basal ganglia activity: relationship to the pathophysiology of Parkinson's disease. Mov Disord 18:357-363

Brown P, Oliviero A, Mazzone P, Insola A, Tonali P, Di LV (2001) Dopamine dependency of oscillations between subthalamic nucleus and pallidum in Parkinson's disease. J Neurosci 21:1033-1038

Conde H, Dormont JF, Farin D (1998) The role of the pedunculopontine tegmental nucleus in relation to conditioned motor performance in the cat. II. Effects of reversible inactivation by intracerebral microinjections. Exp Brain Res 121:411-418

Dickson DW, Rademakers R, Hutton ML (2007) Progressive supranuclear palsy: pathology and genetics. Brain Pathol 17:74-82

Dormont JF, Conde H, Farin D (1998) The role of the pedunculopontine tegmental nucleus in relation to conditioned motor performance in the cat. I. Context-dependent and reinforcement-related single unit activity. Exp Brain Res 121:401-410

Erro E, Gimenez-Amaya JM (1999) Pedunculopontine tegmental nucleus. Anatomy, functional considerations and physiopathological implications. An Sist Sanit Navar 22:189-201

Garcia-Rill E (1991) The pedunculopontine nucleus. Prog Neurobiol 36:363-389

Garcia-Rill E, Homma Y, Skinner RD (2004) Arousal mechanisms related to posture and locomotion: 1. Descending modulation. Prog Brain Res 143:283-290

Halliday DM, Rosenberg JR, Amjad AM, Breeze P, Conway BA, Farmer SF (1995) A framework for the analysis of mixed time series/point process data-theory and application to the study of physiological tremor, single motor unit discharges and electromyograms. Prog Biophys Mol Biol 64:237-278

Hirsch EC, Graybiel AM, Duyckaerts C, Javoy-Agid F (1987) Neuronal loss in the pedunculopontine tegmental nucleus in Parkinson disease and in progressive supranuclear palsy. Proc Natl Acad Sci USA 84:5976-5980

Honda T, Semba K (1995) An ultrastructural study of cholinergic and non-cholinergic neurons in the laterodorsal and pedunculopontine tegmental nuclei in the rat. Neuroscience 68:837-853

Hutchison WD, Allan RJ, Opitz H, Levy R, Dostrovsky JO, Lang AE, Lozano AM (1998) Neurophysiological identification of the subthalamic nucleus in surgery for Parkinson's disease. Ann Neurol 44:622-628

Hutchison WD, Lang AE, Dostrovsky JO, Lozano AM (2003) Pallidal neuronal activity: implications for models of dystonia. Ann Neurol 53:480-488

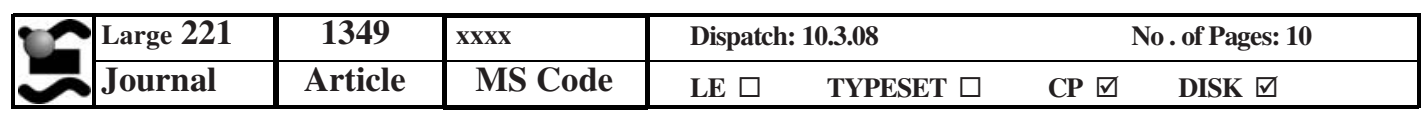


Jarvis MR, Mitra PP (2001) Sampling properties of the spectrum and coherency of sequences of action potentials. Neural Comput 13:717-749

Jellinger K (1988) The pedunculopontine nucleus in Parkinson's disease, progressive supranuclear palsy and Alzheimer's disease. J Neurol Neurosurg Psychiatr 51:540-543

Jenkinson N, Nandi D, Miall RC, Stein JF, Aziz TZ (2004) Pedunculopontine nucleus stimulation improves akinesia in a Parkinsonian monkey. Neuroreport 15:2621-2624

Kaneoke Y, Vitek JL (1996) Burst and oscillation as disparate neuronal properties. J Neurosci Methods 68:211-223

Kleiner-Fisman G, Fisman DN, Sime E, Saint-Cyr JA, Lozano AM, Lang AE (2003) Long-term follow up of bilateral deep brain stimulation of the subthalamic nucleus in patients with advanced Parkinson disease. J Neurosurg 99:489-495

Kobayashi Y, Inoue Y, Yamamoto M, Isa T, Aizawa H (2002) Contribution of pedunculopontine tegmental nucleus neurons to performance of visually guided saccade tasks in monkeys. J Neurophysiol 88:715-731

Kojima J, Yamaji Y, Matsumura M, Nambu A, Inase M, Tokuno H, Takada M, Imai H (1997) Excitotoxic lesions of the pedunculopontine tegmental nucleus produce contralateral hemiparkinsonism in the monkey. Neurosci Lett 226:111-114

Kuhn AA, Trottenberg T, Kivi A, Kupsch A, Schneider GH, Brown P (2005) The relationship between local field potential and neuronal discharge in the subthalamic nucleus of patients with Parkinson's disease. Exp Neurol 194:212-220

Lavoie B, Parent A (1994) Pedunculopontine nucleus in the squirrel monkey: cholinergic and glutamatergic projections to the substantia nigra. J Comp Neurol 344:232-241

Levy R, Hutchison WD, Lozano AM, Dostrovsky JO (2002) Synchronized neuronal discharge in the basal ganglia of parkinsonian patients is limited to oscillatory activity. J Neurosci 22:2855-2861

Matsumura M (2005) The pedunculopontine tegmental nucleus and experimental parkinsonism. A review. J Neurol 252(Suppl 4):IV5-IV12

Matsumura M, Watanabe K, Ohye C (1997) Single-unit activity in the primate nucleus tegmenti pedunculopontinus related to voluntary arm movement. Neurosci Res 28:155-165

Mazzone P, Lozano A, Stanzione P, Galati S, Scarnati E, Peppe A, Stefani A (2005) Implantation of human pedunculopontine nucleus: a safe and clinically relevant target in Parkinson's disease. Neuroreport 16:1877-1881

Mesulam MM, Geula C, Bothwell MA, Hersh LB (1989) Human reticular formation: cholinergic neurons of the pedunculopontine and laterodorsal tegmental nuclei and some cytochemical comparisons to forebrain cholinergic neurons. J Comp Neurol 283:611-633

Olszewsky J, Baxter D (1982) Cytoarchitecture of the human brain stem. Karger, Basale

Pahapill PA, Lozano AM (2000) The pedunculopontine nucleus and Parkinson's disease. Brain 123(Pt 9):1767-1783

Paxinos G, Huang XF (1995) Atlas of the human brainstem. Academic Press, San Diego

Plaha P, Gill SS (2005) Bilateral deep brain stimulation of the pedunculopontine nucleus for Parkinson's disease. Neuroreport 16:1883-1887
Rivlin-Etzion M, Ritov Y, Heimer G, Bergman H, Bar-Gad I (2006) Local shuffling of spike trains boosts the accuracy of spike train spectral analysis. J Neurophysiol 95:3245-3256

Rosenberg JR, Amjad AM, Breeze P, Brillinger DR, Halliday DM (1989) The Fourier approach to the identification of functional coupling between neuronal spike trains. Prog Biophys Mol Biol 53:1-31

Rye DB, Saper CB, Lee HJ, Wainer BH (1987) Pedunculopontine tegmental nucleus of the rat: cytoarchitecture, cytochemistry, and some extrapyramidal connections of the mesopontine tegmentum. J Comp Neurol 259:483-528

Scarnati E, Proia A, Di LS, Pacitti C (1987) The reciprocal electrophysiological influence between the nucleus tegmenti pedunculopontinus and the substantia nigra in normal and decorticated rats. Brain Res 423:116-124

Shink E, Sidibe M, Smith Y (1997) Efferent connections of the internal globus pallidus in the squirrel monkey: II. Topography and synaptic organization of pallidal efferents to the pedunculopontine nucleus. J Comp Neurol 382:348-363

Spann BM, Grofova I (1992) Cholinergic and non-cholinergic neurons in the rat pedunculopontine tegmental nucleus. Anat Embryol (Berl) 186:215-227

Starr PA, Rau GM, Davis V, Marks WJ Jr, Ostrem JL, Simmons D, Lindsey N, Turner RS (2005) Spontaneous pallidal neuronal activity in human dystonia: comparison with Parkinson's disease and normal macaque. J Neurophysiol 93:3165-3176

Stefani A, Lozano AM, Peppe A, Stanzione P, Galati S, Tropepi D, Pierantozzi M, Brusa L, Scarnati E, Mazzone P (2007) Bilateral deep brain stimulation of the pedunculopontine and subthalamic nuclei in severe Parkinson's disease. Brain 130:1596-1607

Takakusaki K, Shiroyama T, Kitai ST (1997) Two types of cholinergic neurons in the rat tegmental pedunculopontine nucleus: electrophysiological and morphological characterization. Neuroscience 79:1089-1109

Tang JK, Moro E, Lozano AM, Lang AE, Hutchison WD, Mahant N, Dostrovsky JO (2005) Firing rates of pallidal neurons are similar in Huntington's and Parkinson's disease patients. Exp Brain Res 166:230-236

Weinberger M, Mahant N, Hutchison WD, Lozano AM, Moro E, Hodaie M, Lang AE, Dostrovsky JO (2006) Beta oscillatory activity in the subthalamic nucleus and its relation to dopaminergic response in Parkinson's disease. J Neurophysiol 96:3248-3256

Winn P (2006) How best to consider the structure and function of the pedunculopontine tegmental nucleus: evidence from animal studies. J Neurol Sci 248:234-250

Yelnik J (2007) PPN or PPD, what is the target for deep brain stimulation in Parkinson's disease? Brain 130:e79

Zrinzo L, Zrinzo LV, Hariz M (2007a) The pedunculopontine and peripeduncular nuclei: a tale of two structures. Brain 130(Pt 6):e73

Zrinzo L, Zrinzo LV, Hariz M (2007b) The peripeduncular nucleus: a novel target for deep brain stimulation? Neuroreport 18:13011302

Zweig RM, Jankel WR, Hedreen JC, Mayeux R, Price DL (1989) The pedunculopontine nucleus in Parkinson's disease. Ann Neurol 26:41-46

\begin{tabular}{|c|c|c|c|c|c|c|}
\hline \begin{tabular}{|l|l|} 
& Large 221
\end{tabular} & 1349 & $\mathbf{x x x x}$ & Dispatc & 0.3 .08 & & . of Pages: 10 \\
\hline Journal & Article & MS Code & LE $\square$ & TYPESET $\square$ & CP च & DISK च \\
\hline
\end{tabular}

